\title{
Autonomie und Autonomieeffekte: Theoretische und methodische Erläuterungen
}

\author{
Falk Ebinger / Carina Schmitt
}

\section{Autonomy and Effects: Explanatory notes on Theory and Methodology}

Abstract: In our article Alles eine Frage des Managements? (Managing for Good?) published in PVS (Ebinger and Schmitt 2010) we presented a concise model to evaluate the explanatory power of factors commonly considered germane to executives' discretion in decision-making and empirically tested it with unique survey data. Bach (2012, in this issue) is puzzled about our theoretical underpinnings, model and case selection. In this article we allay his fears and address pressing theoretical and methodological questions in agencification research.

Keywords: Agencies, Delegation, Performance, Administrative Autonomy, Germany

Schlagwörter: Öffentliche Verwaltung, Verwaltungsreform, Agenturen, Funktionale Politisierung, Performanz

\section{Vorwort}

Hintergrund unseres in der PVS erschienenen Beitrags ist der in zahlreichen Ländern unter dem Paradigma der „Autonomisierung“ vollzogene, grundlegende Verwaltungsumbau hin zu selbstständig(er)en Agencies. Trotz großer Fortschritte der einschlägigen verwaltungswissenschaftlichen Forschung steckt insbesondere der empirische Test möglicher Wirkungsmechanismen zwischen Autonomisierung und besserer Auftragserledigung noch in den Kinderschuhen. Unser Beitrag präsentierte ein Modell, das Autonomie und Entscheidungsverhalten von Führungskräften in Beziehung setzt und so Hinweise auf die Wirkung verschiedener Instrumente der Verselbstständigung gibt. Unser zentrales Argument ist, dass die Ziele von Verselbstständigungen nur erreicht werden können, wenn auf der Mikroebene ein entsprechender Zusammenhang zwischen Autonomie und Entscheidungsverhalten des Leitungspersonals - abgebildet über ihre funktionale Politisierung - besteht (vgl. Ebinger u. Schmitt 2010, S. 74-75). Diese Beziehung sollte sich generell zeigen. Der Untersuchungsfokus wurde deshalb nicht wie so häufig auf explizit verselbstständigte „ideal type agenci$e s$ “ oder sonstige über formale Merkmale definierte Untersuchungseinheiten beschränkt. Auf Basis einer multivariaten Analyse haben wir dieses Modell am Beispiel der Bundesverwaltung einer empirischen Überprüfung unterzogen.

Bachs Kritik an unserem Papier ist vielschichtig. ${ }^{1}$ Wir werden auf alle zentralen Aspekte eingehen. Diese lassen sich wie folgt strukturieren: Bach kritisiert (1) die fehlerhafte Perzeption der Annahmen verschiedener Forschungsstränge, (2) die Unter-

1 Zur Verbesserung der Lesbarkeit wird auf eine wiederholte Nennung des vollständigen Literaturhinweises (Bach 2012) verzichtet und ggf. auf die Seitenzahlen verwiesen. 
schätzung der Bedeutung einzelner Erklärungsfaktoren, insbesondere der formalen Autonomie, und (3) darauf aufbauend eine selektive Fallauswahl und Falleinteilung.

\section{Grundlegende Missverständnisse - Notwendige Klärungen}

Bach beginnt seine Kritik mit der Beobachtung, dass es getrennte Debatten um unabhängige Regulierungsbehörden und managementorientierte Verselbstständigungen im Kontext des NPM gibt. Dies bestreiten wir nicht. Wir interessieren uns für den Mechanismus zwischen Autonomie und den postulierten Erwartungen an eine Verselbstständigung. Wir zielen also ausdrücklich auf die Akteursebene ab, greifen durchweg auf Perzeptionsdaten zurück und wollen Aussagen über Akteursentscheidungen und ihre Determinanten treffen. Dies unterscheidet unseren Ansatz grundlegend von Bachs Bestrebung, mittels Befragungsdaten Aussagen auf institutioneller Ebene zu treffen. Behauptete oder theoretisch konstruierte Ziele hinter der Agenturbildung - Entpolitisierung, Verstetigung und betriebswirtschaftliche Wirkungsorientierung - werden von uns nur insoweit beleuchtet, als sie zur Erhellung dieser Beziehung beitragen (vgl. Ebinger u. Schmitt 2010, S. 73). Tatsächliche Zielsetzungen können von postulierten Zielen abweichen, dennoch müssen sich die so legitimierten Programme an letzteren messen lassen. Aus einer Analyse der Ziele leiten wir unser zentrales Argument eines in der Agenturforschung (d. h. über all ihre Ausdifferenzierungen hinweg) angenommenen Wirkungsmechanismus ab: Professionelleres Verwaltungshandeln soll dadurch erreicht werden, dass , „.... Verwaltungseinheiten jenseits politischer Prärogativen ${ }^{2}$ operativ geführt werden können “ (Ebinger u. Schmitt 2010, S. 75). Unser Kritiker sieht diese generalisierte Aussage als unvereinbar mit den „widersprüchlichen Annahmen“, auf denen die managementorientierte Debatte und die regulierungsorientierten Debatte aufbauen würden (S. 82). So sei im Gegensatz zu Regulierungsbehörden bei den mit Vollzugsaufgaben betrauten Agencies die „Stärkung der politischen Steuerung [...] zentrales Ziel managementorientierter Verwaltungsreformen “ (S. 84). Aus diesem Grund, so Bach, zielten NPM-motivierte Verselbstständigungen nicht auf eine Minimierung der funktionalen Politisierung ab (S. 84).

Tatsächlich werden in den beiden zentralen Diskursen, dem managementorientierten und dem regulierungsorientierten, andere Hauptziele der Verselbstständigung von Behörden angestrebt. Ein Widerspruch liegt jedoch nicht vor, insbesondere nicht auf der von uns betrachteten Akteursebene (vgl. Bach u. Jann 2010, S. 445; Christensen u. Laegreid 2006, S. 8, 13; Döhler 2007, S. 12-13). So soll die erhöhte Steuerungsfähigkeit der Politik als Zielsetzung der managementorientierten Verselbstständigung durch Outputorientierung erreicht werden. Die Agencies sollen nur „[...] indirekt (,at arm's length') über Kontrakte bzw. Zielvereinbarungen gesteuert werden, um so mehr Autonomie für ein effizienzorientiertes Management zu schaffen “ (Döhler 2007, S. 12). Genau um diese operativen Freiheiten der Behördenleitung geht es uns (Ebinger u. Schmitt 2010, S. 73, 75, 76). ${ }^{3}$ Idealtypisch sollten der-

2 Wir meinen explizit Prärogative i. S. v. John Locke als „power to act according to discretion [...]“ und „latitude left to the executive power to do many things of choice which the laws do not prescribe“" (Locke 1823 [1689], Sec. 160).

3 Als Kehrseite einer Verselbstständigung der Leistungserstellung führen wir ausdrücklich die Notwendigkeit verstärkter Leistungskontrolle bei Outputorientierung an: „das größere Maß an Freiheit ginge also mit dem Druck zur Zielerreichung einher" (Ebinger u. Schmitt 2010, S. 73). 
art autonome Einheiten also kaum politisch motivierte Eingriffe ins Tagesgeschäft erleben - ähnlich wie Regulierungsbehörden. ${ }^{4}$ Unser zentrales Argument, dass sich die Wirkung von Verselbstständigungsmaßnahmen bei Regulierungsbehörden und managementorientierten Verselbstständigungen gleicht, bleibt unberührt: Autonomisierung müsste generell mit einer Erweiterung der operativen Handlungsfreiheit einhergehen - und damit mit einer Reduzierung der funktionalen Politisierung.

In einem nächsten Punkt kritisiert Bach, dass wir irrtümlicherweise „in Deutschland empirische Hinweise auf ein ,Agenturfieber“" (S. 85-86) finden. Allerdings ist ein sog. Agenturfieber für die Überprüfung unseres Modells nicht notwendig. Das Modell sollte a priori unabhängig vom Grad der Verselbstständigung universelle Gültigkeit beanspruchen. Bach relativiert seine Kritik jedoch zwei Sätze später unter Rückgriff auf unsere Erläuterungen:

„[...] die Autoren [verweisen] auf die ,lange Geschichte der verselbstständigten Verwaltungseinheiten' (S. 72) in Deutschland, machen aber auch deutlich, dass bestimmte Veränderungen zu beobachten sind, die als Anzeichen einer Zunahme von Agenturen bzw. als vorsichtige Annäherung an das moderne Agenturmodell gedeutet werden (Fußnote 9, S. 78, S. 70)." 5,6

4 Kettls (1997, S. 449) allgegenwärtiger Dualismus betont ausdrücklich die Autonomisierung auf Prozessebene („Letting managers manage“) und die nachgelagerte Kontrolle auf der Outputebene (,Making managers manage“).

5 Jann konstatierte 2004: „Deutschland verfügt auch auf Bundesebene traditionell über viele, sehr unterschiedliche Behörden und Organisationen, die im internationalen Jargon durchaus als Agenturen zu bezeichnen wären“ (2004, S. 107). Und wenige Seiten zuvor (Jann 2004, S. 104): „Mit der dezentralen Ressourcenverantwortung und Budgetierung, die in verschiedenen Bundesbehörden eingeführt wurden, dem Produkthaushalt [...] und internen Leistungsvergleichen [...] wird offenkundig, dass die Bundesverwaltung mit dem nationalen und internationalen Trend schwimmt.“

6 Die anschließende Detailkritik (S. 86-87) halten wir für unbegründet. Da die hier als Belege angeführten Veröffentlichungen bei der Einreichung unseres Papiers noch nicht publiziert waren, lohnt eine Diskussion aber allemal: Bach greift auf eigene Erhebungsdaten zur Zahl abgeschlossener Zielvereinbarungen zurück. Im Gegensatz dazu verwenden wir Daten aus der BT-Drs. 15/5111, da unsere Daten aus demselben Jahr wie die BT-Drs. stammen, seine Daten zum Zeitpunkt der Einreichung unseres Papiers noch nicht erschienen waren und wir hier aus grundsätzlichen und spezifischen methodischen Erwägungen offizielle Zahlen vorziehen. Bach zieht weiterhin in Zweifel, ob man von einem „häufige[n] Abschluss von Zielvereinbarungen zwischen Ministerien und nachgeordneten Behörden" sprechen könne. Angesichts des sich aus den offiziellen Zahlen ergebenden Durchdringungsgrads von 47,3 \% halten wir dies für angemessen. Der Vorwurf, wir würden fälschlicher Weise „von der Anzahl der abgeschlossenen Zielvereinbarungen [...] auf deren Relevanz für die Steuerung der Behörden“ (S. 86) schließen, stimmt bedingt - und zwar insofern, als wir aus der Verbreitung dieses Instrumentariums inter alia auf eine „große[n] Spanne an Organisations- und Steuerungsformen " (Ebinger und Schmitt 2010, S. 72) schließen. Schließlich halten wir es auch immer noch für möglich, dass sich die Verwaltungskultur durch die genannten Trends verändert haben könnte (vgl. Ebinger u. Jochheim 2009). Bach kann weder belegen, dass „die externe Rekrutierung von Führungspersonal“" (Ebinger u. Schmitt 2010, S. 77-78) nur in Einzelfällen vorkommt, noch dass diese nicht doch die Kultur der Behörden prägen. Die von Bach zitierte unveröffentlichte Bachelorarbeit, nach der nur $5 \%$ von 41 Behördenleitern eine „unmittelbare[n] Vorposition in der Privatwirtschaft" (S. 86, Fn. 8) innehatten, halten wir für durchaus glaubwürdig. Der Ausschluss des Führungspersonals der zweiten Ebene und die Begrenzung auf die Privatwirtschaft sind für unsere Fragestellung jedoch unangemessen. Beispielsweise machten ausgerechnet in dem von Bach als Beleg aufgegriffenen Bundesinstitut für Arzneimittel und Medizinprodukte alle früheren und heutigen Leiter und stellvertretenden Leiter lange Jahre Karriere in der Wissenschaft oder als Klinikärzte. Nach der einschlägigen Definition Derliens (2008, S. 304-310) müssten sie folglich durchweg als „Mischtypen“ mit einer verwaltungsfremden Sozialisierung bezeichnet werden. 


\section{Die Bedeutung von Rechtsform und legal autonomy als Explanans}

Unser Kritiker argumentiert, wir hätten auf Basis falscher Annahmen die Rechtsform verselbstständigter Behörden aus unserem Erklärungsmodell ausgeschlossen (S. 87-90). Wir wollen diesen Vorwurf detailliert diskutieren und systematisch aufarbeiten. Sollte Bach Recht haben, würde unserem Modell eine potenziell wichtige erklärende Variable fehlen.

Hintergrund von Bachs Kritik ist eine Fußnote, in der wir den Ausschluss zweier in der Literatur ${ }^{7}$ genannter Variablen, der legal und der structural autonomy begründen. Zur hier vornehmlich relevanten legal autonomy 8 äußern wir uns dabei folgendermaßen (Ebinger u. Schmitt 2010, S. 76, Fußnote 7):

„Legal autonomy erfasst die aus der Rechtsform einer Verwaltungseinheit erwachsende Stabilität. Da die Wahl der Rechtsformen in der bundesdeutschen Verwaltung von einem hohen Maß an Willkür gekennzeichnet und mit keinen besonderen Autonomierechten verbunden ist (vgl. Döhler 2007b, S. 27-28), kann diese Dimension ebenfalls keinen Beitrag zur Erklärung der abhängigen Variable leisten.“

Wir teilen hier die Einschätzung von Döhler (2007, S. 27-28): „De facto verliert jedoch die Rechtsform zunehmend an Aussagekraft, da immer mehr Durchmischungen zu beobachten sind [...]. “9 Bachs Argument (S. 87-90), wir würden hier aufgrund einer Vermischung der Phase der legislativen Steuerung (der Rechtsformenwahl) mit jener der prozeduralen Steuerung (der ministeriellen Steuerungspraxis) ungerechtfertigter Weise aus dem „vergleichsweise große Entscheidungsspielraum bei der Festlegung der Rechtsform" auf eine geringe Bedeutung dieses Faktors schließen, mag formal richtig sein; für unser Modell ist und bleibt die Rechtsform - wie wir zeigen werden - irrelevant. Wir werden im Folgenden unsere Begründung des Ausschlusses der Rechtsform konkretisieren, indem wir belegen, dass (1) die Rechtsform für unserem Modell keine zentrale Rolle spielt, da es sich um eine vorgelagerte Variable handelt, (2) die Erklärungskraft der Rechtsform in den angeführten empirischen Befunden durchaus begrenzt ist und (3) die Rechtsform als Indikator der legal autonomy im deutschen Fall ohnehin problematisch erscheint.

In der Agenturforschung wird üblicherweise zwischen (1) Autonomiedimensionen (vgl. Fn. 7) und (2) Ausprägungen dieser Dimensionen, den Autonomietypen unterschieden. Letztere spiegeln die früh erkannte Notwendigkeit wieder, analy-

7 Verhoest et al. (2004b, S. 105-106) prägten eine gängige Differenzierung der Autonomiedimensionen in management autonomy, policy autonomy, structural autonomy, financial autonomy, legal autonomy und interventional autonomy.

8 Das Konstrukt der legal autonomy entstammt dem grundlegenden (auch von Bach zitierten) Aufsatz von Verhoest. Hier wird ein möglicher kausaler Zusammenhang zwischen Rechtsform als erklärender Variable und dem erklärten Konstrukt legal autonomy i. S. des Schutzes vor „recentralisation of decision-making competencies“ (Verhoest et al. 2004b, S. 106) postuliert, wobei damit nur ein grundsätzlicher Entzug von Kompetenzen, nicht die einzelfallbezogene Intervention gemeint ist.

9 Vgl. ähnlich Bach u. Jann (2010, S. 450): „Yet in contrast to standard assumptions about the well-ordered public sector in Germany, legal categories often remain ambiguous and flexible. [...] Thus, classifying agencies (or any other type of public organization or 'quango') by formal autonomy has potential drawbacks, as the agencies' [...] 'actual level of autonomy' (Bouckaert and Peters, 2004: 24) may not correspond to their formal autonomy". 
tisch zwischen formaler, tatsächlicher („factual“ oder „real“) und wahrgenommener (,perceived“) Autonomie zu differenzieren (vgl. Verhoest et al. 2004a, S. 16 mit Verweisen).

Bachs erste Feststellung (S. 89), „[d]ie Rechtsform [habe] [...] erheblichen Einfluss auf verschiedene Dimensionen formaler Autonomie verselbstständigter Behörden und zieh[e] deutliche ,Konsequenzen für das Steuerungsinstrumentarium (Döhler 2005, S. 222) nach sich " ist offenkundig und wird in unserem Beitrag auch keineswegs in Abrede gestellt. ${ }^{10}$ Es liegt nahe, dass sich die Rechtsform in der formalen, explizit durch "formal regulations, laws and contract documents" (Verhoest et al. 2004a, S. 16) festgeschriebenen Autonomie niederschlägt. Wir setzen allerdings mit dem von uns vorgestellten Modell explizit erst auf der Stufe der wahrgenommenen Autonomie als unabhängiger Variable an. Wir überprüfen die Wirkung der wahrgenommen Autonomie auf die Wahrnehmung genereller Entscheidungsfreiheit ${ }^{11}$ - eben der funktionalen Politisierung (vgl. Ebinger u. Schmitt 2010, S. 75-78). Der Effekt der Rechtsform wäre - soweit vorhanden - also bereits in die von uns berücksichtigten Autonomiedimensionen eingegangen.

Eine berechtigte Frage ist sicherlich, ob wir durch den Ausschluss der wahrgenommenen legal autonomy eine wichtige Determinante unserer abhängigen Variablen auslassen. Um dies beantworten zu können, wäre erst zu prüfen, ob die Rechtsform i. S. der von Bach (S. 89) angenommenen abnehmenden Regelbindung „von der mittelbaren Verwaltung über die unmittelbare Verwaltung, die Bundesprivatverwaltung und die Bundesverrichtungsverwaltung" generell und im deutschen Fall ein valider Indikator für die legal autonomy ist. Bachs Ausführungen zu sich häufenden Befreiungen in der Anwendung des öffentlichen Personal- und Haushaltsrechts in Behörden der mittelbaren Verwaltung scheinen dem zu widersprechen. ${ }^{12}$ Die staatsorganisationsrechtliche Literatur verneint dies ebenso. ${ }^{13}$

Bach selbst scheint die Rechtsform jedoch nicht als Indikator, sondern als eine erklärende Variable für verschiedene Dimensionen formaler Autonomie zu behan-

10 Unser Kritiker weitet den von Verhoest et al. (2004b) postulierten Zusammenhang beträchtlich über das Konstrukt der legal autonomy hinaus aus.

11 Vgl. Operationalisierungen im Anhang von Ebinger u. Schmitt 2010.

12 Bach (S. 89): „Während die Behörden und Einrichtungen der unmittelbaren Bundesverwaltung das öffentliche Personal- und Haushaltsrecht zwingend anwenden müssen, kann deren Anwendung in der mittelbaren Bundesverwaltung gesetzlich eingeschränkt oder aufgehoben werden.“ Die mit Verweis auf Döhler (2007, S. 20) konstatierte Flucht aus der unmittelbaren Bundesverwaltung in rechtsfähige Anstalten scheint indes nicht von Erfolg gekrönt zu sein. Bach u. Jann (2010, S. 459) stellen für die deutschen „Agencies“ fest: „[...] the data suggest a somewhat higher degree of perceived 'red tape' in terms of financial management among indirect agencies." Und (2010, S. 461): „We find no statistically significant differences between the frequency of hierarchical interventions towards direct and indirect agencies (ANOVA)."

13 Art. 86 GG fasst bundeseigene und bundesmittelbare Verwaltung zusammen. Das Grundgesetz etabliert hiermit grundsätzlich „,das Modell einer prinzipiell umfassenden exekutivistischen Organisationsgewalt, unter dem Vorbehalt eines parlamentarischen Zugriffsrechts“ (Burgi 2010a, Art. 86, RN 58). Dieses Zugriffsrecht umfasst die instrumentelle und die organisatorische Ebene, Anhaltspunkte für einen „Verwaltungsvorbehalt" in der einen oder anderen Form finden sich nicht (Burgi 2010a, Art. 86, RN 68ff.). Eine Ministerialfreiheit findet sich nur bei Gewährung von Selbstverwaltungsrechten im Bereich der mittelbaren Bundesverwaltung (Burgi 2010b, Art. 87, RN 109). Die Differenzierung in bundeseigene bzw. bundesmittelbare Verwaltung erscheint damit im deutschen Fall nur bedingt geeignet zur Bestimmung der legal autonomy i.S. des Schutzes vor Kompetenzentzug. 
deln (S. 89). Die gleiche Rolle als Explanans soll die Rechtsform auch für die faktische (bzw. wahrgenommene) Autonomie einnehmen (S. 89): ${ }^{14}$ „Der Einfluss der Rechtsform auf die tatsächliche Autonomie verselbstständigter Behörden konnte in zahlreichen empirischen Studien nachgewiesen werden [...]. "15 Konkret stützt sich Bach bei seiner Aussage, „dass Behörden mit eigener Rechtspersönlichkeit [...] über eine größere Management-Autonomie als Behörden der unmittelbaren Bundesverwaltung (z. B. Bundesoberbehörden) verfügen“ (S. 89) auf empirische Arbeiten von Verhoest et al. (2004b), Bach (2010) sowie Bach u. Huber (i. E.). ${ }^{16}$ Verhoest et al. (2004b) verwenden die Rechtsform als erklärende Variable für verschiedene Autonomiedimensionen in flandrischen Behörden. Dabei stellen sie zwar fest, dass der angenommene Autonomiezuwachs bei größerer hierarchischer Distanz vom Regierungszentrum „more or less supported“ für - und nur für - die Dimension der Management-Autonomie ist (Verhoest et al. 2004b, S. 111). Aufgrund der „substantial heterogeneity of autonomy among organisations with the same legal-formal status" relativieren die Autoren die Aussagekraft dieses Befundes jedoch umgehend (S. 114). In ihrem Abstract stellen Verhoest et al. dann auch klar (Verhoest et al. 2004b, S. 101): “The empirical material points at the dangers of using formal-legal status of a public organisation as an indicator of its autonomy [...]”. Bach (2010) schließlich stellt in seinem Beitrag „Policy and Management Autonomy of Federal Agencies in Germany“ zwar einen „erheblichen Einfluss[es]“ der Rechtsform ${ }^{17}$ - zumindest auf die Management-Autonomie fest. Allerdings ist der Befund nur auf eine Teildimension der Management-Autonomie - der Financial Management Autonomy begrenzt (Bach 2010, S. 104). Für die zwei anderen Teildimensionen der Management Autonomie sowie für Policy Autonomie kann Bach keinen solchen Einfluss nachweisen. Da Bach nur jene Variablen in der multivariaten Analyse berücksichtigt, die zuvor in einer bivariaten Matrix statistisch signifikante Korrelationen mit der abhängigen Variablen aufwiesen, werden zudem theoretische und methodische Fragen aufgeworfen.

Schließlich kommt Bach (S. 89) zu dem Schluss, dass wir durch die fehlende Berücksichtigung der Rechtsform die „graduelle Abnahme hierarchisch-bürokratischer Regelbindung “ (Döhler 2007, S. 20) ignorieren. In unserem Beitrag überprüfen wir allerdings mithilfe von Interaktionstermen explizit, wie sich die Wirkung der Erklärungsfaktoren mit der Nähe zum politischen Zentrum verändert (vgl. Ebinger u. Schmitt 2010, S. 78-79, 84-87).

14 Die als „weitere Dimension der formalen Autonomie“ (S. 89) eingeführte hierarchische Aufsicht ist sicher ein wichtiger Autonomie-Indikator; In unserem Modell ist er auf Ebene der wahrgenommenen Autonomie approximativ über den Indikator Häufigkeit hierarchischer Interventionen erfasst.

15 Ein Blick in die von Bach als Beleg angeführte eigene Literatur weist hingegen auf eine bisher eher schwache empirische Bestätigung eines Zusammenhangs zwischen formaler und wahrgenommenerAutonomie hin (Bach 2010, S. 106): „A number of empirical studies show that organizations with a higher level of formal autonomy do not necessarily perceive higher levels of autonomy (Verhoest et al. 2004a; Laegreid et al. 2006; Yesilkagit and van Thiel 2008)."

16 Bach (2010) war zum Zeitpunkt der Einreichung unseres Beitrages noch nicht erschienen; Bach und Huber ist laut Homepage für 2012 angekündigt.

17 Das Sample ist auf unter hierarchischer Kontrolle der Bundesministerien stehende Einheiten der unmittelbaren und mittelbaren Bundesverwaltung begrenzt. 
Als Fazit dieser Debatte bleibt festzuhalten, dass (1) eine große Formenwahlfreiheit auf Ebene der Bundesverwaltung zu bestehen scheint, aus der auf theoretischer Ebene keine tatsächlichen Steuerungsfolgen abgeleitet werden können, (2) der Zusammenhang der Rechtsform mit verschiedenen Autonomiearten und -dimensionen noch konzeptionell unklar und nicht belastbar empirisch belegt scheint und (3) die Rechtsform für unser Modell nicht von Relevanz ist.

\section{Unvergleichbares vergleichen? Sample und Subgruppen}

Schließlich sieht Bach eine weitere Schwäche unseres Beitrags in der Fallauswahl, da wir explizit nur solche Agencies aufgenommen haben, „deren Aufgabenbereich zumindest potentiell einen politisch relevanten Entscheidungsspielraum aufweist" (Ebinger u. Schmitt 2010, S. 79). Dadurch sei „systematische Verzerrung“ zu erwarten, da nicht nachvollziehbar sei, auf welcher Grundlage die Fallauswahl und die Bestimmung der Subgruppen erfolge (S. 90).

Wie bereits eingangs erwähnt, müsste sich der von uns untersuchte Zusammenhang zwischen wahrgenommener Autonomie und der berichteten funktionalen Politisierung grundsätzlich für alle Behörden und öffentliche Aufgaben vollziehende Einrichtungen nachweisen lassen. Nur wenn dies der Fall ist, können die durch eine Verselbständigung angestrebten Änderungen im Entscheidungsverhalten und damit potentiell Performanzverbesserungen erreicht werden. Optimal wäre folglich eine Vollerhebung über das Kontinuum der mit öffentlichen Aufgaben betrauten Organisationen hinweg. ${ }^{18}$ Aus forschungspragmatischen Gründen erhoben wir lediglich Daten eines Subsamples, wählten dieses aber anhand seiner besonderen politikwissenschaftlichen Relevanz aus. Dass ein starker Zusammenhang zwischen der Autonomie nicht im politischen Interesse stehender Behörden und der funktionalen Politisierung ihres Leitungspersonals bestehen könnte, bestreiten wir nicht, wir halten es sogar für sehr wahrscheinlich. So könnte bspw. die inhaltliche Arbeit des Deutschen Wetterdienstes ${ }^{19}$ politische Bedeutung erlangen und Ziel einer Intervention werden. Allerdings halten wir die Wahrscheinlichkeit dafür für ebenso gering, wie die funktionale Politisierung ihres Leitungspersonals.

Sehr viel spannender ist die Frage, ob der angenommene Zusammenhang zwischen Autonomie und funktionaler Politisierung auch bei jenen Behörden auftritt, die tatsächlich im Fokus der Politik stehen bzw. deren Aufgaben mit einiger Wahrscheinlichkeit politische Salienz erlangen können. Als Vorbild einer solchen Selek-

18 Die Rechtsform i.S. einer Dichotomie von bundeseigener und bundesmittelbarer Verwaltung erscheint auch hier kein adäquates Differenzierungskriterium. Die bundeseigene Verwaltung (unmittelbare Bundesverwaltung) umfasst formal „Obere Bundesbehörden, öffentliche Bundeseinrichtungen, Sondervermögen, Bundesbetriebe und neuere Organisationsformen (Beiräte, Projektgruppen, Bundesbeauftragten und pluralistische Entscheidungsgremien)“ (Burgi 2010a, Art. 86, RN 47, 48). Die Gruppe der bundesunmittelbaren Körperschaften und Anstalten (bundesmittelbare Verwaltung) wäre als „beispielhafte Aufzählung für die Verwendung selbständiger, dem Bund zuzurechnender Rechtsträger zu verstehen [...]“, da privatrechtliche Organisationformen nicht durchgängig verboten seien und Stiftungen d.ö.R. sich wesentlich nur in der Herkunft der verwalteten Vermögenswerte von Anstalten entschieden (Burgi 2010a, Art. 86, RN 52; 2010b, Art. 87, RN 107).

19 Der Deutsche Wetterdienst ist eine bundesunmittelbare, teilrechtsfähige Anstalt des öffentlichen Rechts im Geschäftsbereich des Bundesministeriums für Verkehr, Bau und Stadtentwicklung und müsste dementsprechend in dem von Bach gewählten Sample enthalten sein. 
tion diente Döhler (2002, S. 104), der grob 40 Bundesoberbehörden in den Blick nimmt, da ",only this type of authority performs functions, including regulation, that have the chance to enter the sphere of politics [...]" Wir greifen diesen Ansatz auf, modifizieren den Kreis der uns interessierenden Behörden jedoch. Wir gehen erstens nicht davon aus, dass alle Bundesoberbehörden grundsätzlich dieses Potential besitzen. ${ }^{20}$ Zweitens nehmen wir an, dass auch ausgewählte Einheiten der mittelbaren Bundesverwaltung gute Chancen haben, mit ihren Aufgaben politische Salienz zu erlangen. Deshalb wählten wir über einen Positionsansatz (vgl. Herzog 1982, S. 98-106; Schwanke u. Ebinger 2006, S. 228-232) das Führungspersonal von 51 Agencies aus, deren Betätigungsfeld von einschlägigen Experten (Verwaltungswissenschaftlern und in der Bundesverwaltung tätigen Praktikern (vgl. Ebinger u. Schmitt 2010, S. 79) das Potential zugesprochen wurde, Gegenstand von politischen Kontroversen in Parlament oder Öffentlichkeit zu werden. Betont werden muss, dass wir unser Sample explizit nicht wie (S. 90) behauptet, anhand „extremer Ausprägungen der abhängigen Variablen“ selektieren, sondern anhand der unabhängigen Variablen der politischen Salienz der Behördenaufgabe. Eine solche Auswahl zieht keine Inferenzprobleme und keinen selection bias nach sich, wenn - wie von uns praktiziert - die Auswahlvariable auch als unabhängige Variable in die Untersuchung eingeht (vgl. King et al. 1994, S. 137 wie auch die von Bach zitierten Collier u. Mahoney 1996, S. 60). ${ }^{21}$

Außerdem sollte eine Ausweitung des Samples und die damit einhergehende größere Varianz der politischen Salienz vermutlich wie von Bach prognostiziert zu größeren Effektstärken führen. Wir ziehen jedoch einen konservativeren Test des von uns fokussierten Zusammenhangs vor.

Die Differenzierung zwischen gewöhnlichen „abhängigen Bundesoberbehörden “ und „unabhängigen Bundesoberbehörden“ erfolgte aufgrund der formalen oder informalen Freiheiten, die der zweiten Gruppe zugestanden werden. Bei den „unabhängigen Bundesoberbehörden“ handelt es sich um Regulierungsbehörden oder um Behörden, für die aufgrund ihrer besonderen wissenschaftlichen oder politischen Aufgabe explizit oder implizit eine eingeschränkte inhaltliche Steuerung besteht bzw. vermutet werden kann. ${ }^{22}$ Diese Subgruppe verbindet folglich die Annahme, dass von Seiten relevanter Akteure der politischen Arena ein Interesse an der Minimierung ihrer funktionalen Politisierung besteht.

20 Döhler (2002, S. 118-119) spricht selbst davon, dass gewisse Bundesoberbehörden „unimportant service functions" wahrnehmen.

21 King et al. (1994, S. 137) arbeiteten diese Problematik für qualitative Forschungsdesigns auf: „[...] our selection procedure does not predetermine the outcome [...], since we have not restricted the degree of possible variation in the dependent variable." Collier u. Mahoney (1996, S. 60) betonen: „The key insight for understanding these consequences is the fact that under many circumstances, choosing observations so as to constrain variation on the dependent variable tends to reduce the slope estimate produced by regression analysis, whereas an equivalent mode of selection on the explanatory variable does not."

22 Als „unabhängigen Bundesoberbehörden“ werden klassifiziert: Bundesnetzagentur, Bundeskartellamt, Patent- und Markenamt, Bundesanstalt für Finanzdienstleistungsaufsicht, Bundesinstitut für Risikobewertung, Umweltbundesamt, Bundesinstitut für Arzneimittel und Medizinprodukte, Bundeszentrale für politische Bildung, Bundesbeauftragter für Stasi-Unterlagen, Bundesrepublik Deutschland Finanzagentur GmbH und Gesellschaft für Technische Zusammenarbeit (mittlerweile in der Gesellschaft für Internationale Zusammenarbeit [GIZ] aufgegangen). 
Ob die Bildung zweier Subgruppen innerhalb des nicht-ministeriellen Behördensamples sich als sinnvoll erweist, betrachten wir als eine empirische Frage. Unsere Auswertung stützt die Belastbarkeit unserer Abgrenzung. Die Subgruppe der „unabhängigen Bundesoberbehörden “ weist tatsächlich eine geringere funktionale Politisierung auf als die „abhängigen Bundesoberbehörden“. Wir konnten zeigen, dass der Behördentyp einen eigenständigen und von den anderen erklärenden Variablen unabhängigen Effekt auf die funktionale Politisierung aufweist. Forschungsbemühungen zu den Gründen für diesen interessanten empirischen Befund wären sicherlich vielversprechend. ${ }^{23}$

\section{Fazit}

Das von uns vorgeschlagene Modell eröffnet eine Möglichkeit, die Problematik fehlender vergleichbarer Outputdaten aller verwaltungsübergreifender Vergleiche zu überwinden und die Annahmen von Modellen zur Verwaltungsoptimierung zu testen. Dies gelingt, indem wir auf der Mikroebene die Autonomiewahrnehmung der zentralen Akteure mit den von ihnen gewählten Handlungsoptionen verbinden. Unter Rückgriff auf ein umfassendes Survey der bundesdeutschen Verwaltung konnten durch den Vergleich von Akteursgruppen auf Ministerial- und Bundesverwaltungsebene die Effekte von Verselbständigungen selbst im eher durch mäßige Modernisierung gekennzeichneten bundesdeutschen Kontext untersucht werden. Unser Beitrag zeigt ebenso wie eine jüngst erschienene Studie von Egeberg u. Trondal (2009) ${ }^{24}$ die Chancen eines solchen Ansatzes. Unsere empirische Überprüfung macht deutlich, dass einzig die Politische Salienz und die Policy Autonomie einen systematischen und stabilen Effekt auf die Funktionale Politisierung der bundesdeutschen politisch-administrativen Elite ausübt. Die insbesondere im NPM-orientierten Modernisierungsdiskurs dominante Management Autonomie scheint das Entscheidungsverhalten des Leitungspersonals hingegen weniger zu beeinflussen. Mit unserem Beitrag möchten wir ein Schlaglicht auf die moderne verwaltungswissenschaftliche Debatte setzen, die theoriegeleitet, methodisch anspruchsvoll und eingebunden in die internationale Public Administration Forschung komplexe Fragestellung mit hoher praktischer Bedeutung bearbeitet. Dieses Feld an der Schnittstelle zwischen Politik-, Wirtschafts- und Rechtswissenschaften erscheint gerade in Deutschland unterbelichtet und verdient nicht nur in Zeiten der Krise mehr Aufmerksamkeit.

23 Selbstverständlich müssen wir uns für die verdienstvolle Suche nach offensichtlichen Fehlern bedanken: Ein working paper von Christensen und Lægreid 2005 fiel den Platzbeschränkungen zum Opfer; das daraus entnommene Zitat (welches sich tatsächlich in Christensen u. Lægreid 2005, S. 5 , findet) auf S. 71 unseres Beitrages entging jedoch der Tilgung und wurde fälschlicherweise Greve et al. 1999 zugewiesen (vgl. Bach S. 81, Fn. 4). Letztere schreiben zwar sinngemäß dasselbe, aber eben nicht wörtlich. Außerdem sind im Anhang unseres Artikels tatsächlich nicht explizit alle, sondern aus Platzgründen nur jene Einheiten aufgelistet, die mit einer Antwort in die Auswertung eingingen. Die Grundgesamtheit unserer Befragung umfasste 51 Agencies, wir erzielten einen Rücklauf aus 40 Agencies oder 78,4 Prozent. Die Grundgesamtheit und detaillierte Daten zum Rücklauf finden Interessierte in einem frühen, auf der EGPA Conference 2007 in Madrid der Fachöffentlichkeit präsentierten Entwurf unseres Beitrags (Ebinger 2007).

24 Die Studie erschien leider erst nach Einreichung unseres Beitrages. 


\section{Literatur}

Bach, Tobias. 2010. Policy and management autonomy of federal agencies in Germany. In Governance of Public Sector Organizations. Proliferation, Autonomy and Performance, Hrsg. Per Laegreid und Koen Verhoest, 89-110. Hampshire: Palgrave Macmillan.

Bach, Tobias. 2012. Autonomie und Steuerung verselbstständigter Behörden: Management, Regulierung und die Bedeutung formaler Strukturen. Politische Vierteljahresschrift 53:XX-XX.

Bach, Tobias, und Etienne Huber. 2012. Comparing Agencification in Continental Countries. In Government Agencies: Practices and Lessons from 30 Countries, Hrsg. Koen Verhoest, Sandra van Thiel, Geert Bouckaert und Per Laegreid. Basingstoke: Palgrave Macmillan (im Erscheinen).

Bach, Tobias, und Werner Jann. 2010. Animals in the administrative zoo: organizational change and agency autonomy in Germany. International Review of Administrative Sciences 76:443-468.

Burgi, Martin. 2010a. Kommentierung von Art. 86 GG „Bundeseigene Verwaltung“. In Das Bonner Grundgesetz, Kommentar, Hrsg. Hermann v. Mangoldt, Friedrich Klein und Christian Starck, Band 3, 6. Aufl., 109-204. München: Verlag Vahlen.

Burgi, Martin. 2010b. Kommentierung von Art. 87 GG „Gegenstände der bundeseigenen Verwaltung“. In Das Bonner Grundgesetz, Kommentar, Hrsg. Hermann v. Mangoldt, Friedrich Klein und Christian Starck, Band 3, 6. Aufl., 131-188. München: Verlag Vahlen.

Christensen, Tom, und Per Laegreid. 2005. Agencification and Regulatory Reforms. Konferenzpapier für den SCANCOR/SOG workshop on "Automization of the state: From integrated administrative models to single purpose organizations”. Stanford 1-2.4.2005.

Christensen, Tom, und Per Laegreid. 2006. Agencification and Regulatory Reforms. In Autonomy and Regulation: Coping with Agencies in the Modern State, Hrsg. Tom Christensen und Per Laegreid, 8-49. Cheltenham: Edward Elgar.

Collier, David, und James Mahoney. 1996. Insights and Pitfalls. Selection Bias in Qualitative Research. World Politics 49:56-91.

Derlien, Hans-Ulrich. 2008. Die politische und die administrative Elite der Bundesrepublik. In Regieren zu Beginn des 21. Jahrhunderts, Hrsg. Werner Jann und Klaus König, 291328. Tübingen: Mohr Siebeck.

Döhler, Marian. 2002. Institutional Choice and Bureaucratic Autonomy in Germany. West European Politics 25:101-124.

Döhler, Marian. 2005. Die begrenzte Rationalität von Delegation und Steuerung in der Bundesverwaltung. In Mechanismen der Politik, Hrsg. Steffen Ganghof und Philip Manow, 215-243. Frankfurt a. M.: Campus Verlag.

Döhler, Marian. 2007. Vom Amt zur Agentur? Organisationsvielfalt, Anpassungsdruck und institutionelle Wandlungsprozesse im deutschen Verwaltungsmodell. In Agencies in Westeuropa, Hrsg. Werner Jann und Marian Döhler, 12-47. Wiesbaden: VS Verlag für Sozialwissenschaften.

Ebinger, Falk. 2007. Agencies and Effects. Autonomy and Functional Politicization in German Federal Agencies. Konferenzpapier präsentiert auf der European Group of Public Administration (EGPA) Annual Conference, Permanent Study Group VI - Governance of Public Sector Organizations, Madrid, 19-22.9.2007. http://soc.kuleuven.be/io/egpa/ org/2007madrid/papers/paper_ebinger.pdf. Zugegriffen: 7.11.2011. 
Ebinger, Falk, und Linda Jochheim. 2009. Wessen loyale Diener? Wie die große Koalition die deutsche Ministerialbürokratie veränderte. dms - der moderne staat 2:335-353.

Ebinger, Falk, und Carina Schmitt. 2010. Alles eine Frage des Managements? Wie Autonomierechte die Handlungsfreiheit des administrativen Führungspersonals beeinflussen. Politische Vierteljahresschrift 51:69-93.

Egeberg, Morten, und Jarle Trondal. 2009. Political Leadership and Bureaucratic Autonomy: Effects of Agencification. Governance 22:673-688.

Greve, Carsten, Matthew Flinders und Sandra Van Thiel. 1999. Quangos - What's in a name? Defining Quangos from a Comparative Perspective. Governance 12:129-146.

Herzog, Dietrich. 1982. Politische Führungsgruppen. Probleme und Ergebnisse der modernen Elitenforschung. Darmstadt: Wissenschaftliche Buchgesellschaft.

Jann, Werner. 2004. Verwaltungsmodernisierung auf Bundesebene. In Status-Report Verwaltungsreform. Eine Zwischenbilanz nach zehn Jahren, Hrsg. Werner Jann, Jörg Bogumil, Geert Bouckaert, Dietrich Budäus, Lars Holtkamp, Leo Kissler, Sabine Kuhlmann, Christoph Reichard und Hellmut Wollmann, 100-112. Berlin: edition sigma.

Kettl, Donald F. 1997. The Global Revolution in Public Management: Driving Themes, Missing Links. Journal of Policy Analysis and Management 16:446-462.

King, Gary, Robert O. Keohane und Sidney Verba 1994. Designing Social Inquiry. Scientific Inference in Qualitative Research. Princeton: Princeton University Press.

Locke, John. 1823 [1689]. Two Treatises of Government. A New Edition, Corrected. Prepared by Rod Hay for the McMaster University Archive of the History of Economic Thought. http://www.efm.bris.ac.uk/het/locke/government.pdf. Zugegriffen: 18.12.2011.

Schwanke, Katja, und Falk Ebinger. 2006. Politisierung und Rollenverständnis der deutschen Administrativen Elite 1970 bis 2005. Wandel trotz Kontinuität. In Politik und Verwaltung, Politische Vierteljahresschrift Sonderheft 37, Hrsg. Jörg Bogumil, Werner Jann und Frank Nullmeier, 228-246. Wiesbaden: VS Verlag für Sozialwissenschaften.

Verhoest, Koen, B. Guy Peters, Geert Bouckaert und Bram Verschuere. 2004a. The Study of Organisational Autonomy: A Conceptual Review. Public Administration and Development 24:101-118.

Verhoest, Koen, B. Guy Peters, Geert Bouckaert und Bram Verschuere. 2004b. The Study of Organisational Autonomy: A Conceptual and Methodological Review. http://soc. kuleuven.be/sbov. Zugegriffen: 10.7.2005.

\section{Autorenangaben:}

Dipl. Verw.-Wiss. Falk Ebinger,

Ruhr Universität Bochum, Fakultät für Sozialwissenschaft, Lehrstuhl Öffentliche Verwaltung, Stadt- und Regionalpolitik, Universitätsstraße 150, 44801 Bochum, falk.ebinger@rub.de

[Korrespondenzautor]

Dr. Carina Schmitt, Universität Bremen, Center for Social Policy Research, Mary-Sommerville-Straße 5, 28359 Bremen carina.schmitt@sfb597.uni-bremen.de 